Article

\title{
Three Scrophularia Species (Scrophularia buergeriana, S. koraiensis, and S. takesimensis) Inhibit RANKL-Induced Osteoclast Differentiation in Bone Marrow-Derived Macrophages
}

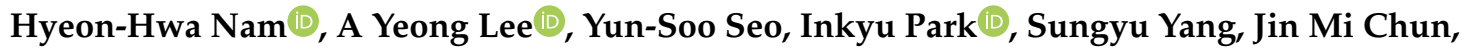 \\ Byeong Cheol Moon $\mathbb{D}^{\mathrm{D}}$, Jun-Ho Song * $\mathbb{D}$ and Joong-Sun Kim * $\mathbb{D}$ \\ Herbal Medicine Resources Research Center, Korea Institute of Oriental Medicine, 111, Geonjae-ro, \\ Naju-si 58245, Korea; hhnam@kiom.re.kr (H.-H.N.); lay7709@kiom.re.kr (A.Y.L.); sys0109@kiom.re.kr (Y.-S.S.); \\ pik6885@kiom.re.kr (I.P.); sgyang81@kiom.re.kr (S.Y.); jmchun@kiom.re.kr (J.M.C.); bcmoon@kiom.re.kr (B.C.M.) \\ * Correspondence: songjh@kiom.re.kr (J.-H.S.); centraline@kiom.re.kr (J.-S.K.)
}

Received: 20 October 2020; Accepted: 24 November 2020; Published: 26 November 2020

\begin{abstract}
Scrophulariae Radix, derived from the dried roots of Scrophularia ningpoensis Hemsl. or S. buergeriana Miq, is a traditional herbal medicine used in Asia to treat rheumatism, arthritis, and pharyngalgia. However, the effects of Scrophularia buergeriana, S. koraeinsis, and S. takesimensis on osteoclast formation and bone resorption remain unclear. In this study, we investigated the morphological characteristics and harpagoside content of S. buergeriana, S. koraiensis, and S. takesimensis, and compared the effects of ethanol extracts of these species using nuclear factor (NF)- $\kappa B$ ligand (RANKL)-mediated osteoclast differentiation. The harpagoside content of the three Scrophularia species was analyzed by high-performance liquid chromatography-mass spectrometry (HPLC/MS). Their therapeutic effects were evaluated by tartrate-resistant acid phosphatase (TRAP)-positive cell formation and bone resorption in bone marrow-derived macrophages (BMMs) harvested from ICR mice. We confirmed the presence of harpagoside in the Scrophularia species. The harpagoside content of S. buergeriana, S. koraiensis, and S. takesimensis was $1.94 \pm 0.24 \mathrm{mg} / \mathrm{g}, 6.47 \pm 0.02 \mathrm{mg} / \mathrm{g}$, and $5.50 \pm 0.02 \mathrm{mg} / \mathrm{g}$, respectively. Treatment of BMMs with extracts of the three Scrophularia species inhibited TRAP-positive cell formation in a dose-dependent manner. The area of hydroxyapatite-absorbed osteoclasts was markedly decreased after treatment with the three Scrophularia species extracts. Our results indicated that the three species of the genus Scrophularia might exert preventive effects on bone disorders by inhibiting osteoclast differentiation and bone resorption, suggesting that these species may have medicinal and functional value.
\end{abstract}

Keywords: Scrophularia buergeriana; S. koraiensis; S. takesimensis; harpagoside; osteoclast differentiation; RANKL

\section{Introduction}

An imbalance between osteoclasts and osteoblasts affects bone formation, leading to weakened bone and the development of skeletal diseases such as osteoporosis, rheumatoid arthritis, lytic bone metastases, and chronic obstructive pulmonary disease. The function of osteoclasts, multinucleated giant cells, is bone resorption, but osteoporosis can occur if bone resorption exceeds formation due to an increase in the number of osteoclasts [1]. Most drugs used to treat osteoporosis inhibit osteoclast differentiation to control bone resorption. Receptor activator of nuclear factor (NF)- $\mathrm{KB}$ ligand (RANKL), a major osteoclastogenic molecule, is a member of the tumor necrosis factor (TNF) superfamily and is the initial stimulator of osteoclast differentiation, inducing the expression of osteoclast-associated 
genes, such as tartrate-resistant acid phosphatase (TRAP) [2,3]. Therefore, bisphosphonates and anti-RANKL antibodies that inhibit osteoclast activity are currently used for the treatment of bone resorption diseases.

Scrophulariae Radix, an herbal medicine known as Korean Hyun-Sam, is derived from the dried roots of Scrophularia ningpoensis Hemsl. or S. buergeriana Miq., plants which are widely distributed throughout the temperate regions of the Northern Hemisphere, including Asia, Europe, and North America [4-6]. Scrophulariae Radix has been traditionally used as a therapeutic agent for blood cooling, yin nourishing, fire pursing, and toxin removal, and is widely used to treat rheumatism and arthritis in Southwest Asia [7-10]. It has also been reported to have neuroprotective, anti-inflammatory, anti-allergy, anti-amnesia, antioxidant, and hepatoprotective effects [11-15]. In Korea, S. koraiensis Nakai (Korean: To-Hyun-Sam) has been used as an antipyretic and anti-inflammatory agent in traditional medicine. S. takesimensis Nakai (Korean: Seom-Hyun-Sam) is restricted to Ulleung-do Island [16,17]. Although this species is a valuable endemic resource, its medicinal efficacy has not been assessed to date.

The therapeutic potential of Scrophularia species is associated with the functions of major secondary metabolites, such as phenylpropanoids and iridoid glycosides, which are present in the plant $[18,19]$. Harpagoside, an iridoid component present in the Scrophularia species, is a bioactive compound of Harpagophytum procumbens DC. (Devil's Claw) and has been used in Southern Africa to treat pain, arthritis, and ulcers. Pharmacological effects of harpagoside on RANKL-induced osteoclast differentiation have also been reported [20,21]. However, there are few reports concerning the pharmacological activity of Scrophularia species on RANKL-induced osteoclast differentiation, and studies on the biological activity of $S$. koraiensis and $S$. takesimensis have not been reported.

In the current study, we compared the morphological characteristics and harpagoside content of S. buergeriana, S koraiensis, and S. takesimensis, and compared the effects of Scrophularia species extracts on RANKL-mediated osteoclast differentiation.

\section{Results}

\subsection{Comparative Morphology of Scrophularia Species}

The three species can be distinguished on the basis of leaf shape, apex, margins, pubescence of stems, and calyx shape (Table 1). The leaf blade of S. buergeriana is ovate, with an acute apex (Figure 1A), and serrate with a spinose tooth marginal shape (Figure 1D). The stem is glabrous (Figure 1G), and the calyx is ovate with an obtuse apex (Figure 1J). S. koraiensis has lanceolate to rarely ovate-shaped leaf blades with an acuminate apex (Figure 1B) and is serrate with a spinose tooth (Figure 1E). The stem of S. koraiensis is sparsely pubescent with non-glandular trichomes (Figure $1 \mathrm{H}$ ), and the calyx is lanceolate with an acute to attenuate apex (Figure $1 \mathrm{~K}$ ). However, S. takesimensis has ovate leaf blades with an acute apex (Figure 1C), and has a serrate, almost without spinose tooth, marginal leaf blade (Figure 1F). The stem surface of $S$. takesimensis is glabrous (Figure 1I), and its calyx is semicircular, with a rounded apex (Figure 1L).

Table 1. Major determinants of Scrophularia species.

\begin{tabular}{|c|c|c|c|c|c|c|}
\hline & \multicolumn{3}{|c|}{ Leaves } & \multirow{2}{*}{$\begin{array}{c}\text { Stems } \\
\text { Surfaces }\end{array}$} & \multicolumn{2}{|c|}{ Calyx } \\
\hline & Shape & Apex & Margins & & Shape & Apex \\
\hline S. buergeriana & ovate & acute & $\begin{array}{l}\text { serrate with } \\
\text { spinose tooth }\end{array}$ & glabrous & ovate & obtuse \\
\hline S. koraiensis & $\begin{array}{c}\text { lanceolate to } \\
\text { rarely ovate }\end{array}$ & acuminate & $\begin{array}{l}\text { serrate with } \\
\text { spinose tooth }\end{array}$ & pubescent & lanceolate & $\begin{array}{l}\text { acute to } \\
\text { attenuate }\end{array}$ \\
\hline S. takesimensis & ovate & acute & $\begin{array}{l}\text { serrate almost } \\
\text { without spinose tooth }\end{array}$ & glabrous & semi-circular & rounded \\
\hline
\end{tabular}



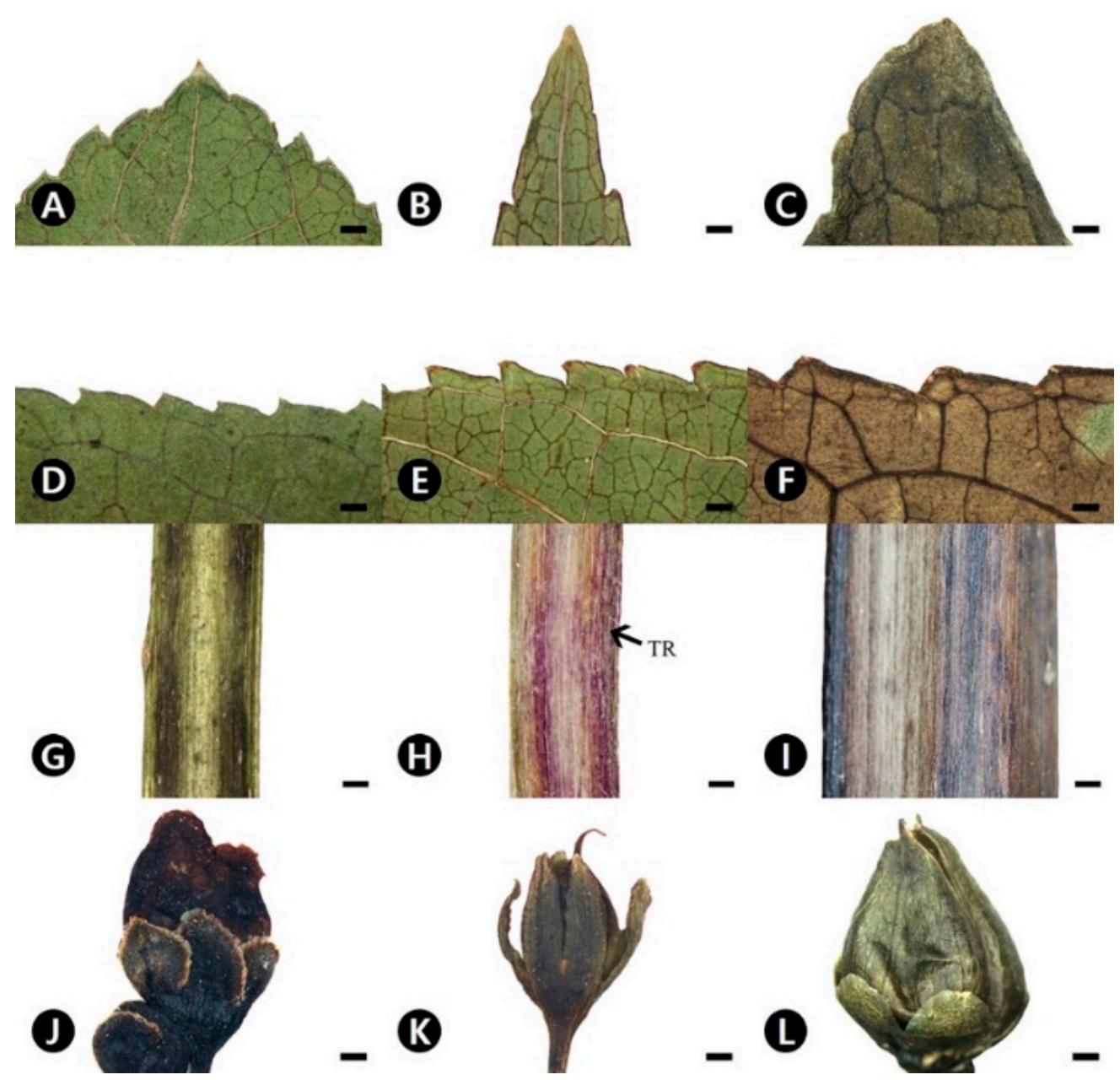

Figure 1. Stereomicroscope micrographs showing the morphology of the three Scrophularia species studied. (A-C) Apex of leaf blade. (D-F) Margin of leaf blade. (G-I) Surface of stem. (J-L) Calyx of flower and/or fruit. (A,D,G,J) S. buergeriana. (B,E,H,K) S. koraiensis. (C, F,I,L) S. takesimensis. TR, Trichomes. All scale bars $=1 \mathrm{~mm}$.

\subsection{Harpagoside Content of Scrophulariae Species}

High-performance liquid chromatography (HPLC) chromatograms of S. buergeriana, S. koraiensis, and S. takesimensis are shown in Figure 2A,B. Figure 2A shows the HPLC chromatograms of the harpagoside standard compound and the three species of Scrophulariae monitored at $280 \mathrm{~nm}$, because the maximum wavelength of harpagoside is $279.5 \mathrm{~nm}$. Harpagoside was detected at approximately $50.1 \mathrm{~min}$. The harpagoside content of S. buergeriana, S. koraiensis, and S. takesimensis was $1.94 \pm 0.24 \mathrm{mg} / \mathrm{g}$, $6.47 \pm 0.02 \mathrm{mg} / \mathrm{g}, 5.50 \pm 0.02 \mathrm{mg} / \mathrm{g}$, respectively (Table 2). The total ion chromatography (TIC) of the mass spectrometry (MS) spectrum was confirmed from 190-850 $\mathrm{m} / \mathrm{z}$, and the extracted ion chromatogram (XIC) of harpagoside in the samples was analyzed at $517.11 \mathrm{~m} / \mathrm{z}$, because harpagoside was detected at $517.11 \mathrm{~m} / z[\mathrm{M}-\mathrm{H}+\mathrm{Na}]^{+}$(Figure 2B). 
(A)
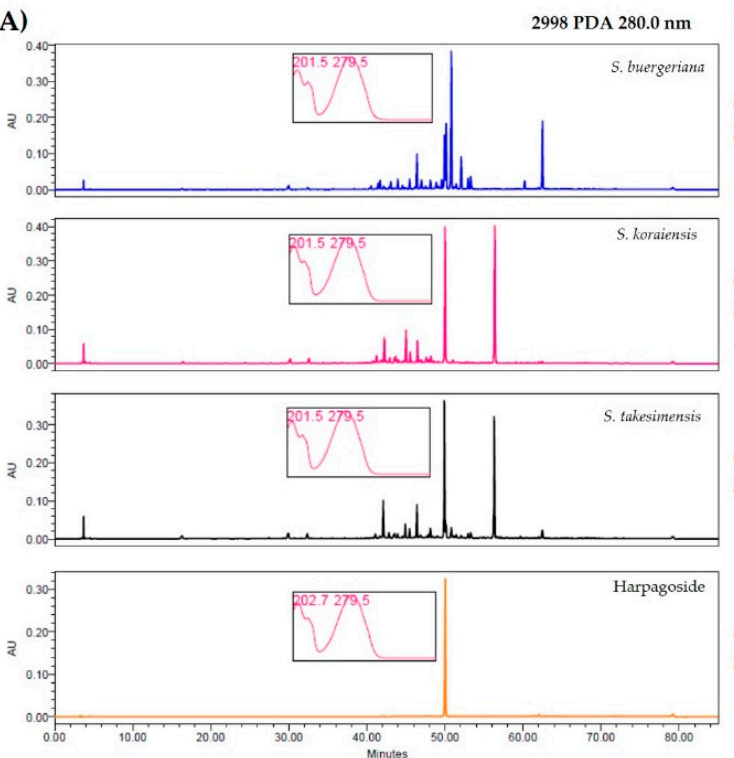

(B)
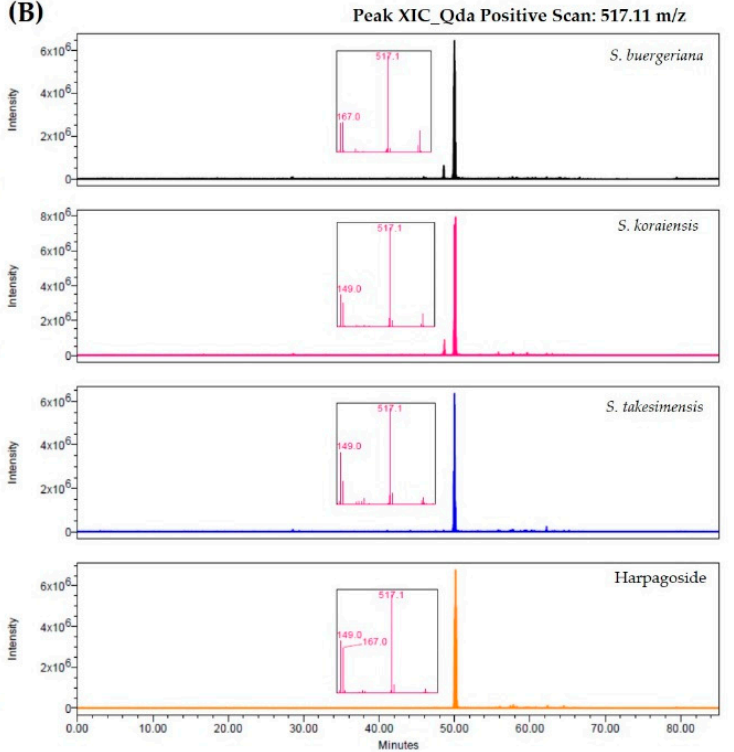

Figure 2. (A) Chromatograms of three Scrophularia species (S. buergeriana, S. koraiensis, and S. takesimensis) at $280 \mathrm{~nm}$ for harpagoside $\left(\lambda_{\max }=279.5 \mathrm{~nm}\right)$ and $(\mathbf{B})$ positive extracted ion chromatogram $(\mathrm{XIC})$ spectrum of harpagoside at $517.11 \mathrm{~m} / \mathrm{z}$.

Table 2. Harpagoside content of three Scrophularia species (S. buergeriana, S. koraiensis, and S. takesimensis) by high-performance liquid chromatography (HPLC) chromatogram analysis.

\begin{tabular}{cc}
\hline Scrophulariae Species & Harpagoside $(\mathbf{m g} / \mathbf{g})$ \\
\hline S. buergeriana & $1.94 \pm 0.24(1)$ \\
S. koraiensis & $6.47 \pm 0.02$ \\
S. takesimensis & $5.50 \pm 0.02$ \\
\hline
\end{tabular}

(1) Values are expressed as means \pm standard deviation (SD) of three samples for each species.

\subsection{Cytotoxic Effects on Primary Murine BMM Growth}

The XTT assay was conducted to assess cytotoxicity during osteoclast differentiation. S. buergeriana, S. koraiensis, and S. takesimensis did not reduce cell viability at most of the concentrations tested. Treatment with $200 \mu \mathrm{g} / \mathrm{mL}$ S. koraiensis reduced cell viability from that of the normal control group (Figure 3A-C), but the difference was not statistically significant.
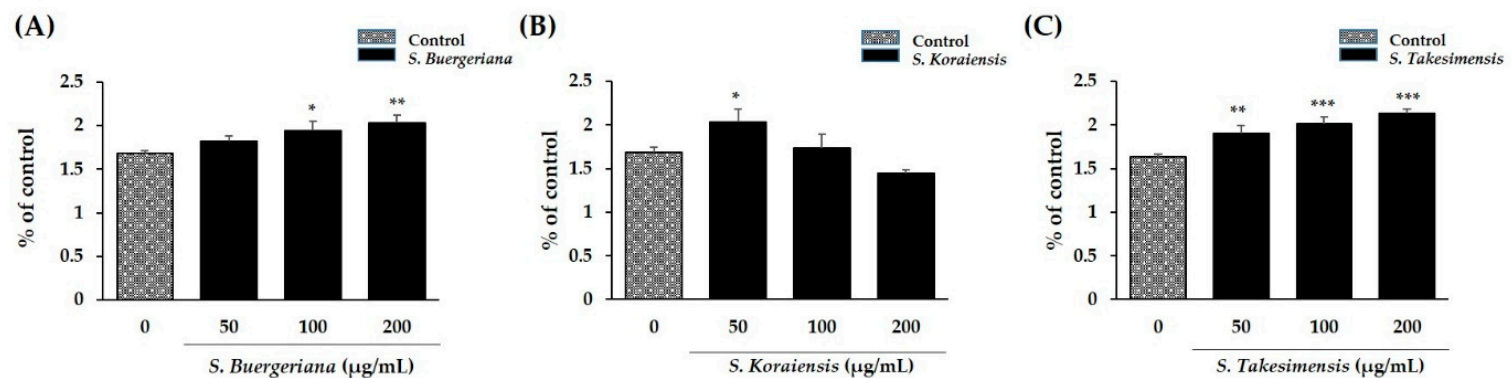

Figure 3. Cell viability affected by ethanol extracts of the three Scrophularia species (A) S. buergeriana (B) S. koraiensis and (C) S. takesimensis. ${ }^{*} p<0.05,{ }^{* *} p<0.01$, and ${ }^{* * *} p<0.001$ vs. control (dimethyl sulfoxide; DMSO).

\subsection{Effects on Osteoclast Differentiation}

To compare the effect of S. buergeriana, S. koraiensis, and S. takesimensis on osteoclast differentiation, mouse BMMs treated with macrophage colony stimulating factor (M-CSF) and RANKL were cultured 
in the presence or absence of ethanol extracts of the three Scrophularia species. RANKL and M-CSF induced differentiation of BMMs after incubation for 4 days. TRAP-positive osteoclasts were present in higher numbers in the control group, whereas treatment with Scrophularia species extracts inhibited the formation of TRAP-positive cells in a dose-dependent manner (Figure 4B). The S. koraiensis treatment group showed suppressed formation of RANKL-induced TRAP activity at concentrations of $100 \mu \mathrm{g} / \mathrm{mL}$ and $200 \mu \mathrm{g} / \mathrm{mL}$.

(A)

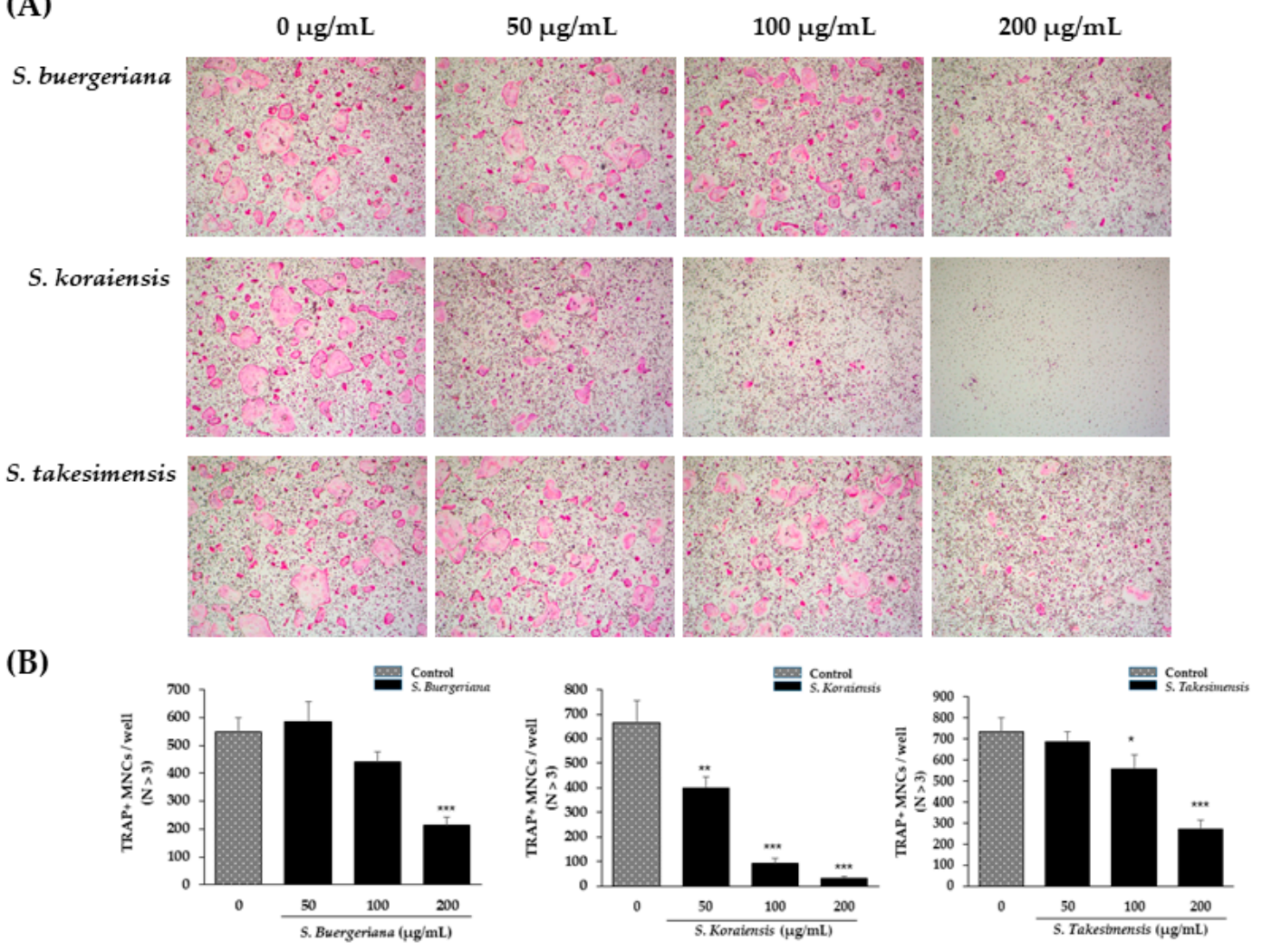

Figure 4. Effects on osteoclast differentiation of ethanol extracts of Scrophularia buergeriana, S. koraiensis, and S. takesimensis at concentrations of $50 \mu \mathrm{g} / \mathrm{mL}, 100 \mu \mathrm{g} / \mathrm{mL}$, and $200 \mu \mathrm{g} / \mathrm{mL}$. (A) Tartrate-resistant acid phosphatase (TRAP)-positive cells photographed (100 $\times$ magnification) after bone marrow macrophages were cultured with macrophage colony stimulating factor (M-CSF) and nuclear factor (NF)- $\mathrm{B}$ ligand (RANKL) in the presence of ethanol extracts of the three Scrophularia species. (B) TRAP-positive cells were counted as osteoclasts. ${ }^{*} p<0.05,{ }^{* *} p<0.01$, and ${ }^{* * *} p<0.001$ vs. control (DMSO).

\subsection{Effects on Bone Resorption}

To evaluate the effects of Scrophularia species on bone resorption, mature osteoclasts and extracts of S. buergeriana, S. koraiensis, and S. takesimensis were applied to plates coated with hydroxyapatite for symbiotic culture of the osteoblasts. Although the area of hydroxyapatite-adsorbed osteoclasts was increased in the control, the resorption area was markedly decreased by treatment with extracts of the three Scrophularia species. The resorption inhibition effect was highest for S. buergeriana, followed by S. koraiensis, and then S. takesimensis. (Figure 5A,B). 
(A)

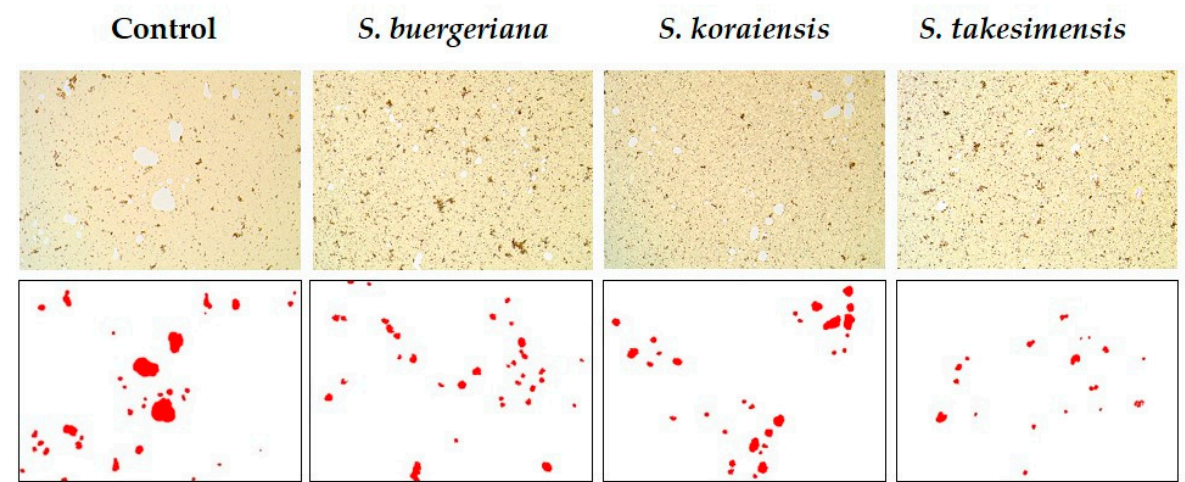

(B)
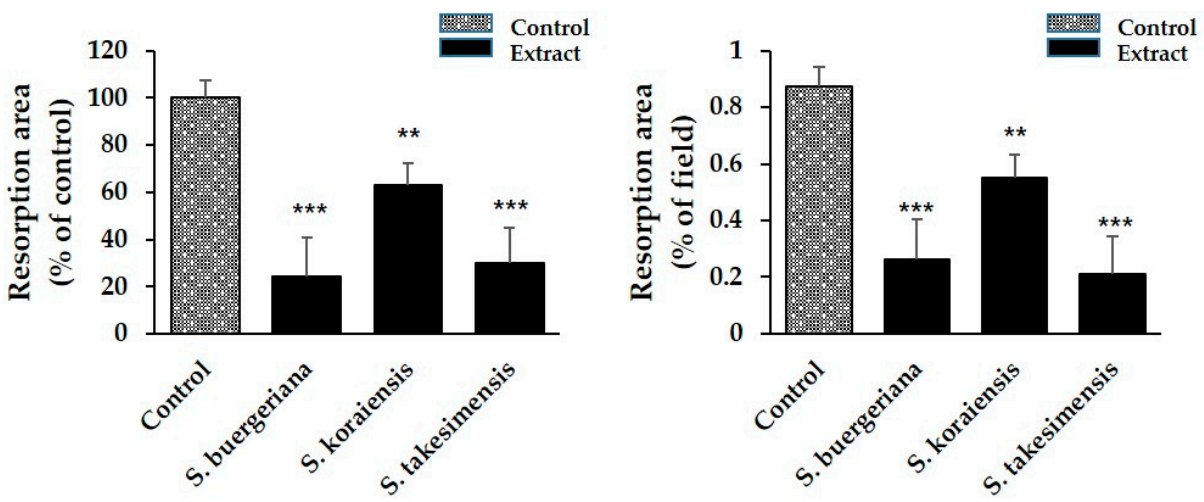

Figure 5. Effects of S. buergeriana, S. koraiensis, and S. takesimensis on bone resorption by mature osteoclasts. (A) Hydroxypatite-adherent cells were collected and imaged under a light microscope. (B) Resorption areas quantified on hydroxyapatite-coated plates. ${ }^{* *} p<0.01$ and ${ }^{* *} p<0.001$ vs. control (DMSO).

\section{Discussion}

Osteoporosis, one of the major diseases attracting attention worldwide, is associated with lowered bone mass density as a result of an imbalance between the osteoblasts and osteoclasts that influence bone homeostasis $[22,23]$. Therapeutic agents such as bisphosphonates are currently used to treat bone resorption diseases, but the long-term use of these drugs leads to the suppression of bone formation and osteonecrosis [24]. Therefore, the beneficial pharmacological effects of plant-derived natural compounds have been advocated [10].

Several studies have reported that iridoid glycosides in Scrophularia plant extracts demonstrate anti-inflammatory, neuroinflammation, antioxidant, and hepatoprotective effects [11-13,15,25] Harpagoside, an iridoid glycoside, is a main bioactive component of Harpagophytum procumbens (family Pedaliaceae) root used to treat chronic rheumatism, osteoarthritis, and arthritis, and is an active constituent of Scrophularia species [21,26-28]. We quantified the harpagoside content in S. buergeriana, S. koraiensis, and S. takesimensis using HPLC/MS (Table 2, Figure 2). Harpagoside was detected in three Scrophularia species, with the highest content found in S. koraiensis extract.

In this study, we investigated the inhibitory effects exerted by three Scrophularia species containing harpagoside against osteoclast differentiation and bone resorption without cytotoxic effects in RANKL-induced cells. The cytotoxicity of S. buergeriana, S. koraiensis, and S. takesimensis was evaluated in BMMs and measured by XTT assay during osteoclast differentiation. Treatment with ethanol extracts of the three Scrophularea species did not reveal cytotoxic effects on BMMs up to $200 \mu \mathrm{g} / \mathrm{mL}$, with more than $90 \%$ cell viability being observed.

RANKL, a bone formation biomarker, is associated with stimulation of osteoblasts and osteoclast differentiation [22]. The balance of bone formation is influenced by osteoblasts and bone resorption activity by osteoclastic cells [29]. Previous studies have demonstrated that harpagoside improved 
bone properties by inhibiting the formation of osteoclasts from BMMs and the maturation of osteoblast cells $[21,30]$.

TRAP expression is associated with osteoclast maturation and differentiation and is a standard approach to the detection of osteoclasts [24]. In our study, TRAP staining indicated that the numbers and areas of TRAP-positive cells increased, whereas the S. buergeriana, S. koraiensis, and S. takesimensis treatment groups exhibited considerably fewer TRAP-stained osteoclasts, without any cytotoxicity. The results suggested that the three Scrophularia species inhibit osteoclast differentiation and formation in BMMs. The inhibitory effect of harpagoside was increased in a dose-dependent manner by downregulating TRAP expression in BMCs [30]. Therefore, it is presumed that the difference in efficacy of the three Scrophularia species in our results would be affected by the content of harpagoside. To study the inhibitory effects of Scrophularia species on bone resorption by osteoclast formation induced by RANKL, we investigated bone resorption pit generation in mature osteoclasts. Although the area of hydroxyapatite-absorbed osteoclasts was increased in DMSO controls, the resorption area was markedly decreased by treatment with the three Scrophularia species. The highest resorption inhibition effect was exerted by S. buergeriana, followed by S. takesimensis, and then S. koraiensis.

\section{Materials and Methods}

\subsection{Chemicals}

Harpagoside was purchased from Shanghai Sunny Biotech (Shanghai, China). HPLC-grade solvents were obtained from Merck (Darmstadt, Germany). Sodium 3'-[1-(phenyl-aminocarbonyl)-3,4 -tetrazolium]-bis(4-methoxy-6-nitro) benzene sulfonic acid hydrate (XTT reagent) was purchased from Sigma-Aldrich (St. Louis, MO, USA).

\subsection{Plant Materials}

Samples of the three Scrophularia species (S. buergeriana, S. koraiensis, and S. takesimensis) were obtained from the Department of Herbal Crop Research (Eumseong-gun, Chungcheongbuk-do, Korea). The same vouchers were used for both chemical and morphological analyses (S. buergeriana; 2-18-0144, S. koraiensis; 2-18-0145, S. takesimensis; 2-18-0146) from the Korean Herbarium of Standard Resources (KHSR), Korean Institute of Oriental Medicine.

\subsection{Comparative Morphology of Scrophularia Species}

S. buergeriana and S. koraiensis were originally collected from the experimental field of the National Institute of Horticultural and Herbal Science (NIHHS, Korea), and S. takesimensis was collected from the natural population on Ulleung-do Island. Vouchers of the studied medicinal materials (S. buergeriana (2-18-0144), S. koraiensis (2-18-0145), and S. takesimensis (2-18-016)) were deposited in the Korean Herbarium of Standard Herbal Resources (Index Herbariorum code: KIOM) at the Korea Institute of Oriental Medicine (Naju, Korea). We observed the voucher specimens of the three species (Figure S1) sing an Olympus SZX16 stereomicroscope (Olympus, Tokyo, Japan) for morphological comparison.

\subsection{HPLC/MS Analysis}

S. buergeriana $(778.14 \mathrm{~g})$, S. koraiensis $(69.15 \mathrm{~g})$, and S. takesimensis $(92.5 \mathrm{~g})$ were refluxed in $70 \%$ ethanol $(v / v)$ for $2 \mathrm{~h}$, and these extracts were filtered through filter paper and evaporated in vacuo. The powders of the $70 \%$ ethanol extract for S. buergeriana, S. koraiensis, and S. takesimensis were $363.83 \mathrm{~g}$ $(46.76 \%$ of yield, $w / w), 30.40 \mathrm{~g}(43.96 \%$ of yield, $w / w)$, and $53.91 \mathrm{~g}(58.28 \%$ of yield, $w / w)$, respectively, and these extracts were stored at $4{ }^{\circ} \mathrm{C}$. Accurately weighed powders of three $70 \%$ ethanol extracts (10 mg) were dissolved in $10 \mathrm{~mL} \mathrm{70 \%} \mathrm{ethanol} \mathrm{and} \mathrm{filtered} \mathrm{through} \mathrm{a} 0.2 \mu \mathrm{m}$ syringe filter prior to HPLC analysis. HPLC was performed using a Waters e2695 Separation Module (Waters Corporation, Milford, MA, USA) and a 2998 photodiode array detector (Waters), combined with an Acquity QDa detector and a micro-splitter (IDEX Health \& Science LLC, Oak Harbor, WA, USA) for MS. A Kinetex 
Phenyl-hexyl 100A column $(4.6 \times 250 \mathrm{~mm}, 5 \mu \mathrm{m}$, Phenomenex Inc., Torrance, CA, USA) was utilized. The mobile phase was $0.05 \%$ aqueous formic acid (A), methanol (B), and acetonitrile (C) using the following gradient program: $100 \% \mathrm{~A}(2 \mathrm{~min}) \rightarrow 96 \% \mathrm{~A}(3 \% \mathrm{~B}$ and $1 \% \mathrm{C}, 7 \mathrm{~min}) \rightarrow$ isocratic $85 \% \mathrm{~A}$ $(11 \% \mathrm{~B}$ and $4 \% \mathrm{C}$, from $15 \mathrm{~min}$ to $20 \mathrm{~min}) \rightarrow 70 \% \mathrm{~A}(23 \% \mathrm{~B}$ and $7 \% \mathrm{C}, 35 \mathrm{~min}) \rightarrow 50 \% \mathrm{~A}(35 \% \mathrm{~B}$ and $15 \%$ C, $45 \mathrm{~min}) \rightarrow 30 \%$ A (50\% B and $20 \%$ C, $55 \mathrm{~min}$ ). The following conditions were applied: The flow rate was $0.8 \mathrm{~mL} / \mathrm{min}$, injected volume was $10 \mu \mathrm{L}$, and UV wavelength was monitored from $195 \mathrm{~nm}$ to $400 \mathrm{~nm}$. The QDa mass detector employed the following conditions: Nitrogen carrier gas, $0.8 \mathrm{kV}$ electrospray ionization (ESI) capillary, $600{ }^{\circ} \mathrm{C}$ probe temperature, $15 \mathrm{~V}$ con voltage, $120^{\circ} \mathrm{C}$ source temperature, 20:1 split. TIC was monitored from $150 \mathrm{~m} / \mathrm{z}$ to $850 \mathrm{~m} / \mathrm{z}$. Harpagoside was detected at $280 \mathrm{~nm}$ and $517.11 \mathrm{~m} / \mathrm{z}$.

\subsection{BMM (Bone Marrow Macrophage) Isolation and Culture}

Five-week-old ICR mice were sacrificed by cervical dislocation, the thigh and shin bones were aseptically excised, and the soft tissue was removed. After cutting both ends of the iliac crest, bone marrow cells were obtained by flushing both ends of the bone material using a $1 \mathrm{~mL}$ syringe. The isolated BMMs were incubated in a culture dish for 1 day in $\alpha$-MEM medium containing $10 \%$ FMS and 1\% penicillin/streptomycin, and unsaturated cells were collected. After 3 days, attached macrophages were used in this experiment. Macrophages were treated with M-CSF $(30 \mathrm{ng} / \mathrm{mL})$ and RANKL $(100 \mathrm{ng} / \mathrm{mL})$, and incubated with S. takesimensis, S. koraiensis, and S. buergeriana extracts at concentrations of $50 \mu \mathrm{g} / \mathrm{mL}, 100 \mu \mathrm{g} / \mathrm{mL}$, and $200 \mu \mathrm{g} / \mathrm{mL}$. The day following subculture, the cultured cells were stained with a TRAP solution. Cells with three or more nuclei among red-stained cells were considered differentiated osteoclasts, and the degree of differentiation was measured.

\subsection{Cell Cytotoxicity}

BMMs were seeded in 96-well plates at a density of $1 \times 10^{4}$ cells/well and were treated with M-CSF $(30 \mathrm{ng} / \mathrm{mL}$ ) and $50 \mu \mathrm{g} / \mathrm{mL}, 100 \mu \mathrm{g} / \mathrm{mL}$, and $200 \mu \mathrm{g} / \mathrm{mL}$ ethanol extracts of Scrophularia species (S. takesimensis, S. koraiensis, and S. buergeriana) for 3 days. After 3 days, $50 \mu \mathrm{L}$ XTT reagent (Sigma-Aldrich) was applied for $4 \mathrm{~h}$. The optical density was measured at $450 \mathrm{~nm}$ using an ELISA reader (Biotek Instruments, Winooski, VT, USA).

\subsection{Bone Resorption Pit Assay}

To obtain mature osteoclasts, BMMs obtained from the shin and thigh bones of 5-week-old ICR mice and osteoclasts isolated from the skull of 1-day-old ICR mice were added to a $90 \mathrm{~mm}$ culture plate coated with collagen. To produce a symbiotic culture, $1 \alpha, 25$-Dihydroxyvitamin D3 (VitD3) and prostaglandin E2 were added. After incubation for 6 days, cells were removed by treatment with $0.1 \%$ collagenase and added to a 96 -well plate coated with hydroxyapatite. At the same time, S. takesimensis, S. koraiensis, and S. buergeriana extracts were added to the hydroxyapatite-coated plates at a concentration of $200 \mathrm{ug} / \mathrm{mL}$ and cultured for $24 \mathrm{~h}$. The cells were washed with distilled water and observed through an optical microscope (Nikon, Tokyo, Japan). The hydroxyapatite-absorbed portion was imaged using micrography, and the area was measured using ImageJ software version 1.51 (National Institutes of Health, Bethesda, MD, USA).

\subsection{Statistical Analysis}

Data were expressed as the mean \pm standard deviation (SD). Statistical analysis was performed by analysis of variance (ANOVA), followed by a multiple comparison procedure using Dunnett's test. A value of $p<0.05$ indicated significant difference. 


\section{Conclusions}

Our results suggest that the Scrophularia species may prevent bone loss by inhibiting osteoclast differentiation and born resorption without causing cytotoxicity. It was confirmed that similar metabolites were contained in the Scrophularia species, and their extracts exerted similar efficacy. S. buergeriana and S. koraiensis demonstrated inhibitory effects against osteoclast differentiation and bone resorption and had the highest harpagoside content. Therefore, the three Scrophularia species, including S. koraiensis, may be potentially therapeutic for treating bone disorder diseases, but the mechanism underlying their bioactivity requires further study.

Supplementary Materials: The following are available online at http://www.mdpi.com/2223-7747/9/12/1656/s1.

Author Contributions: Conceptualization, J.-S.K. and J.-H.S.; the morphology study, J.-H.S.; in-vitro analysis, H.-H.N., Y.-S.S. and J.M.C.; resources, I.P. and S.Y.; chemical analysis, A.Y.L.; genetic analysis, B.C.M.; funding acquisition, B.C.M., J.-H.S. and J.-S.K.; writing-review and editing, H.-H.N., J.-H.S. and J.-S.K.; All authors have read and agreed to the published version of the manuscript.

Funding: This research was supported by the Korea Institute of Oriental Medicine, Daejeon, South Korea (grant numbers K18402, KSN2012320) and the National Research Foundation of Korea (NRF) grant funded by the Korea government (MSIT) (NRF-2020R1A2C1004272 and NRF-2020R1A2C1100147).

Acknowledgments: We thank the Korean Herbarium of Standard Herbal Resources (herbarium code KIOM) for the provision of materials. J.-H.S. sincerely thanks Jeong Hoon Lee (Department of Herbal Crop Research, NIHHS) for assistance in material sampling.

Conflicts of Interest: The authors declare no conflict of interest.

\section{Abbreviations}

BMM, bone marrow-derived macrophage; RANKL, receptor activator of nuclear factor (NF)- $\mathrm{kB}$ ligand; TIC, total ion chromatography; TRAP, tartrate-resistant acid phosphatase; XIC, extracted ion chromatogram; XTT, sodium 3'-[1-(phenyl-aminocarbonyl)-3,4-tetrazolium]-bis(4-methoxy-6-nitro) benzene sulfonic acid hydrate.

\section{References}

1. Yu, L.; Jia, D.; Feng, K.; Sun, X.; Xin, H.; Qin, L.; Han, T. A natural compound (LCA) isolated from Litsea cubeba inhibits RANKL-induced osteoclast differentiation by suppressing Akt and MAPK pathways in mouse bone marrow macrophages. J. Ethnopharmacol. 2020, 257, 112873. [CrossRef] [PubMed]

2. Boyle, W.J.; Simonet, W.S.; Lacey, D.L. Osteoclast differentiation and activation. Nature 2003, 423, 337-342. [CrossRef] [PubMed]

3. Lee, C.H.; Kwak, S.C.; Kim, J.Y.; Oh, H.M.; Rho, M.C.; Yoon, K.H.; Yoo, W.H.; Lee, M.S.; Oh, J. Genipin inhibits RANKL-induced osteoclast differentiation through proteasome-mediated degradation of c-Fos protein and suppression of NF-kB activation. J. Pharmacol. Sci. 2014, 124, 344-353. [PubMed]

4. Yamazaki, T. Scrophulariaceae. In Flora of Japan; Iwatsuki, K., Yamazaki, T., Boufford, D.E., Ohba, H., Eds.; Kodansha: Tokyo, Japan, 1993; Volume IIIa, pp. 326-331.

5. Hong, D.Y.; Yang, H.B.; Jin, C.L.; Fischer, M.A.; Holmgren, N.H.; Mill, R.R. Scrophularia. In Flora of China; Wu, Z.Y., Raven, P.H., Eds.; Science Press and Missori Botanical Garden Press: Beijing, China; St. Louis, MO, USA, 1998; Volume 18, pp. 11-20.

6. Choi, H.K. Scrophularia. In The Genera of Vascular Plants of Korea; Flora of Korean Editorial Committee, Ed.; Hongreung Publishing Co.: Seoul, Korea, 2018; pp. 1148-1150.

7. Wang, S.; Hua, Y.; Zou, L.; Liu, X.; Yan, Y.; Zhao, H.; Luo, Y.; Liu, J. Comparison of Chemical Constituents in Scrophulariae Radix Processed by Different Methods based on UFLC-MS Combined with Multivariate Statistical Analysis. J. Chromatogr. Sci. 2017, 56, 122-130. [CrossRef]

8. You-Hua, C.; Jin, Q.; Jing, H.; Bo-Yang, Y. Structural characterization and identification of major constituents in Radix Scrophulariae by HPLC coupled with electrospray ionization quadrupole time-of-flight tandem mass spectrometry. Chin. J. Nat. Med. 2014, 12, 47-54. [CrossRef]

9. Huang, X.Y.; Chen, C.X.; Zhang, X.M.; Liu, Y.; Wu, X.M.; Li, Y.M. Effects of ethanolic extract from Radix Scrophulariae on ventricular remodeling in rats. Phytomedicine 2012, 19, 193-205. [CrossRef] 
10. Zengin, G.; Stefanucci, A.; Rodrigues, M.J.; Mollica, A.; Custodio, L.; Aumeeruddy, M.Z.; Mahomoodally, M.F. Scrophularia lucida L. as a valuable source of bioactive compounds for pharmaceutical applications: In vitro antioxidant, anti-inflammatory, enzyme inhibitory properties, in silico studies, and HPLC profiles. J. Pharm. Biomed. Anal. 2019, 162, 225-233. [CrossRef]

11. Lee, H.J.; Spandidos, D.A.; Tsatsakis, A.; Margina, D.; Izotov, B.N.; Yang, S.H. Neuroprotective effects of Scrophularia buergeriana extract against glutamate-induced toxicity in SH-SY5Y cells. Int. J. Mol. Med. 2019, 43, 2144-2152. [CrossRef]

12. Shin, N.R.; Lee, A.Y.; Song, J.H.; Yang, S.; Park, I.; Lim, J.O.; Jung, T.Y.; Ko, J.W.; Kim, J.C.; Lim, K.S.; et al. Scrophularia buergeriana attenuates allergic inflammation by reducing NF- $\mathrm{KB}$ activation. Phytomedicine 2020, 67, 153159. [CrossRef]

13. Jeong, E.J.; Ma, C.J.; Lee, K.Y.; Kim, S.H.; Sung, S.H.; Kim, Y.C. KD-501, a Standardized Extract of Scrophularia buergeriana Has Both Cognitive-Enhancing and Antioxidant Activities in Mice Given Scopolamine. J. Ethnopharmacol. 2009, 121, 98-105. [CrossRef]

14. Kim, S.R.; Kang, S.Y.; Lee, K.Y.; Kim, S.H.; Markelonis, G.J.; Oh, T.H.; Kim, Y.C. Anti-amnestic Activity of E-p-methoxycinnamic acid from Scrophularia buergeriana. Cogn. Brain Res. 2003, 17, 454-461. [CrossRef]

15. Lee, E.J.; Kim, S.R.; Kim, J.; Kim, Y.C. Hepatoprotective Phenylpropanoids From Scrophularia Buergeriana Roots Against CCl(4)-induced Toxicity: Action Mechanism and Structure-Activity Relationship. Planta Med. 2002, 68, 407-411. [CrossRef] [PubMed]

16. Wang, P.; Wang, D.; Qi, Z.; Li, P.; Fu, C. Scrophularia koraiensis, a new synonym to Scrophularia kakudensis (Lamiales: Scrophulariaceae). Phytotaxa 2015, 202, 228-230. [CrossRef]

17. Ma, S.M.; Lim, Y.S.; Na, S.T.; Lee, J.; Shin, H.C. Genetic structure and population differentiation of endangered Scrophularia takesimensis (Scrophulariaceae) in Ulleung Island, Korea. Korean J. Plant Taxon. 2011, 41, 182-193. [CrossRef]

18. Pasdaran, A.; Hamedi, A. The genus Scrophularia: A source of iridoids and terpenoids with a diverse biological activity. Pharm. Biol. 2017, 55, 2211-2233. [CrossRef] [PubMed]

19. García, D.; Fernández, A.; Sáenz, T.; Ahumada, C. Antiinflammatory effects of different extracts and harpagoside isolated from Scrophularia frutescens L. Farmaco 1996, 51, 443-446. [PubMed]

20. Haseeb, A.; Ansari, M.Y.; Haqqi, T.M. Harpagoside suppresses IL-6 expression in primary human osteoarthritis chondrocytes. J. Orthop. Res. 2017, 35, 311-320. [CrossRef]

21. Kim, J.Y.; Park, S.H.; Baek, J.M.; Erkhembaatar, M.; Kim, M.S.; Yoon, K.H.; Oh, J.; Lee, M.S. Harpagoside inhibits RANKL-induced osteoclastogenesis via Syk-Btk-PLC $\gamma 2-\mathrm{Ca}(2+)$ signaling pathway and prevents inflammation-mediated bone loss. J. Nat. Prod. 2015, 78, 2167-2174. [CrossRef]

22. Zhang, B.; Yang, L.L.; Ding, S.Q.; Liu, J.J.; Dong, Y.H.; Li, Y.T.; Li, N.; Zhao, X.J.; Hu, C.L.; Jiang, Y.; et al. Anti-osteoporotic activity of an edible traditional Chinese medicine Cistanche deserticola on bone metabolism of ovariectomized rats through RANKL/RANK/TRAF6-mediated signaling pathways. Front. Pharmacol. 2019, 10, 1412. [CrossRef]

23. Kim, K.J.; Lee, Y.; Son, S.R.; Lee, H.; Son, Y.J.; Lee, M.K.; Lee, M. Water Extracts of Hull-less Waxy Barley (Hordeum vulgare L.) Cultivar ‘Boseokchal’ Inhibit RANKL-induced Osteoclastogenesis. Molecules 2019, 24, 3735. [CrossRef]

24. Kim, J.H.; Kim, M.; Jung, H.S.; Sohn, Y. Leonurus sibiricus L. ethanol extract promotes osteoblast differentiation and inhibits osteoclast formation. Int. J. Mol. Med. 2019, 44, 913-926. [CrossRef] [PubMed]

25. Meng, X.; Xie, W.; Xu, Q.; Liang, T.; Xu, X.; Sun, G.; Sun, X. Neuroprotective Effects of Radix Scrophulariae on Cerebral Ischemia and Reperfusion Injury via MAPK Pathways. Molecules 2018, 23, 2401. [CrossRef] [PubMed]

26. Che, D.; Cao, J.; Liu, R.; Wang, J.; Hou, Y.; Zhang, T.; Wang, N. Harpagoside-induced anaphylactic reaction in an IgE-independent manner both in vitro and in vivo. Immunopharmacol. Immunotoxicol. 2018, 40, 173-178. [CrossRef]

27. Su, L.; Deng, Y.; Chen, N.; Zhang, X.; Huang, T. Infrared-assisted extraction followed by high performance liquid chromatography to determine angoroside C, cinnamic acid, and harpagoside content in Scrophularia ningpoensis. BMC Complement. Altern. Med. 2019, 19, 130. [CrossRef] [PubMed]

28. Axmann, S.; Hummel, K.; Nöbauer, K.; Razzazi-Fazeli, E.; Zitterl-Eglseer, K. Pharmacokinetics of harpagoside in horses after intragastric administration of a Devil's claw (Harpagophytum procumbens) extract. J. Vet. Pharmacol. Ther. 2019, 42, 37-44. [CrossRef] [PubMed] 
29. Kumagai, M.; Nishikawa, K.; Mishima, T.; Yoshida, I.; Ide, M.; Watanabe, A.; Fujita, K.; Morimoto, Y. Fluorinated Kavalactone Inhibited RANKL-Induced Osteoclast Differentiation of RAW264 Cells. Biol. Pharm. Bull. 2020, 43, 898-903. [CrossRef]

30. Chung, H.J.; Kim, W.K.; Park, H.J.; Cho, L.; Kim, M.R.; Kim, M.J.; Shin, J.S.; Lee, J.H.; Ha, I.H.; Lee, S.K. Anti-osteoporotic activity of harpagide by regulation of bone formation in osteoblast cell culture and ovariectomy-induced bone loss mouse models. J. Ethnopharmacol. 2016, 179, 66-75. [CrossRef]

Publisher's Note: MDPI stays neutral with regard to jurisdictional claims in published maps and institutional affiliations.

(C) 2020 by the authors. Licensee MDPI, Basel, Switzerland. This article is an open access article distributed under the terms and conditions of the Creative Commons Attribution (CC BY) license (http://creativecommons.org/licenses/by/4.0/). 